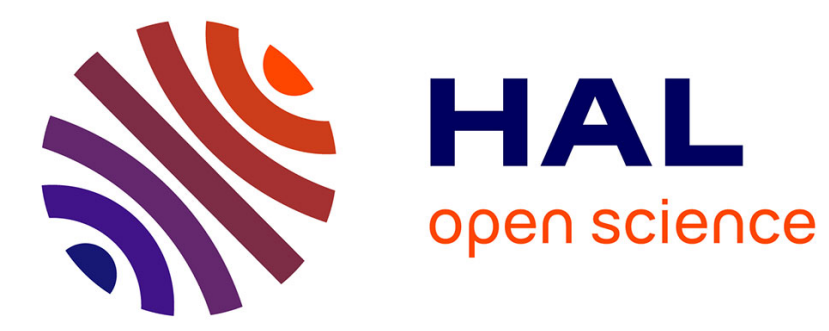

\title{
Connecting the ground state mean square radius to the dipole excitation
}

\author{
R. Mezhoud, F.-Z. Ighezou, R.J. Lombard
}

\section{To cite this version:}

R. Mezhoud, F.-Z. Ighezou, R.J. Lombard. Connecting the ground state mean square radius to the dipole excitation. Physica Scripta, 2007, 75, pp.82-86. 10.1088/0031-8949/75/1/013 . in2p300134827

\section{HAL Id: in2p3-00134827 https://hal.in2p3.fr/in2p3-00134827}

Submitted on 5 Mar 2007

HAL is a multi-disciplinary open access archive for the deposit and dissemination of scientific research documents, whether they are published or not. The documents may come from teaching and research institutions in France or abroad, or from public or private research centers.
L'archive ouverte pluridisciplinaire HAL, est destinée au dépôt et à la diffusion de documents scientifiques de niveau recherche, publiés ou non, émanant des établissements d'enseignement et de recherche français ou étrangers, des laboratoires publics ou privés. 


\title{
Connecting the ground state mean square radius to the dipole excitation.
}

\author{
R. Mezhoud ${ }^{a, b}$, F.-Z. Ighezou ${ }^{a}$ and R.J. Lombard ${ }^{c}$ \\ ${ }^{a}$ Institut de Physique, USTHB \\ Bab Ezzouar, Alger, Algeria \\ ${ }^{b}$ Faculty of Sciences, Boumerdes University, \\ 35000 Boumerdes, Algeria \\ ${ }^{c}$ Groupe de Physique théorique \\ Institut de Physique Nucléaire \\ 91406 Orsay Cedex, France
}

September 20. 2006

\begin{abstract}
We study relationships between the dipole excitation and the ground state ms radius of a two-body system in the case of local potentials. We recall the inequality obtained long ago by Bertlmann and Martin, and discuss correction factors transforming the inequality in an approximate expression. Connecting the correction factor to the contribution of the lowest dipole state to the sum rule, we get a lower bound to the ms radius. Inverting the relationships yields a bound for the square of the dipole transition matrix element, and thus a bound to the lowest dipole state transition rate.
\end{abstract}

PACS : 03.65Ge ; $31.10+z ; 32.70 \mathrm{Cs}$ 
The Bertlmann-Martin inequality [1,2] yields an upper bound to the ground state mean square radius (ms radius) from the lowest dipole excitation energy. It concerns a particle moving in a local potential or two particles interacting via a scalar interaction. This relationship is exact in the case of the harmonic oscillator. A simple correction factor proposed by Bertlmann and Martin [1] extends its exact character to the Coulomb potential. It also constitutes a good approximation for a wide class of potentials [3,4]. First established in $D=3$ dimensional space, this situation is valid in any $D \geq 2$ space [5].

The correction factor proposed by Bertlmann and Martin [1] uses only spectroscopic data, i.e. the energy of the $2 \mathrm{~s}$ state. In this respect it is very efficient. At the same time, it is legitimate to ask the question of a possible alternative, based on another observable. Because the saturation of the inequality is directly connected to the rate of the sum rule exhausted by the lowest excited state, linking the correction factor to this rate is a very natural choice. Thus the purpose of the present work is investigate what can be learned from this rate.

As we shall see, the present study establishes a lower bound to the ground state ms radius in terms of the dipole transition matrix element. Its inversion produces a bound to the transition probability or the lifetime of the lowest dipole state. The correction factor built from the lowest contribution to the dipole sum rule is found to be depending on the potential, but in such a way that it can still be used to improve the lower bound mentioned above.

\section{The dipole excitation case.}

We consider the Schrödinger equation in the $D=3$ dimensional space, for a particle in a local potential, or equivalently two spin-less particles interacting via a scalar interaction. The spherical symmetry is also assumed. In this case, the Bertlmann-Martin bound [1, 2] reads

$$
<r^{2}>\leq \frac{3}{2\left(E_{1,1}-E_{1,0}\right)}
$$

Here, $\left\langle r^{2}\right\rangle$ is the ground state ms radius. Use is made of units $\hbar=m=1$. The states are labeled by $n$ and $\ell$, where $n-1$ is the number of nodes of the wave function, and $\ell$ the angular momentum. The equality is reached for the harmonic oscillator.

The bound (1) is obtained from an under-estimation of the dipole sum rule :

$$
\begin{aligned}
& {\left[E_{1,1}-E_{1,0}\right] \sum_{n=1}^{\infty}|<n, 1| z|1,0>|^{2}} \\
& \quad \leq \sum_{n=1}^{\infty}\left[E_{n, 1}-E_{1,0}\right]|<n, 1| z|1,0>|^{2}=\frac{1}{2} .
\end{aligned}
$$


Here $z=2 \sqrt{\pi / 3} r Y_{1,0}(\theta, \varphi)[6]$. The next step uses the closure $\sum_{n=1}^{\infty}|n, \ell><n, \ell|=1$ in the left hand term of (2), together with $<1,0|z| 1,0\rangle=0$.

Formally, the bound (1) can be transformed into an equality by introducing a correction factor

$$
<r^{2}>=\frac{3}{2\left(E_{1,1}-E_{1,0}\right)} \varphi
$$

Clearly, $\varphi$ depends on the potential, and nothing is gained unless this factor can be reached in a model independent way. Bertlmann and Martin $[1,2]$ have proposed the empirical expression

$$
\varphi \approx 1-\frac{1}{4}\left[\frac{E_{2,0}+E_{1,0}-2 E_{1,1}}{E_{2,0}-E_{1,0}}\right]^{2}
$$

This form is exact for the Coulomb potential and preserves the equality in the harmonic oscillator case. It is an excellent approximation for a wide class of potentials [3], and in particular for the power-law potentials [4]. These ones are defined by

$$
V(r)=\operatorname{sign}(\alpha) V_{0} r^{\alpha} ; \alpha>-2 \text { and } \neq 0
$$

Note that the correction factor (4) loses its efficiency as $\alpha<-1$ and tends to -2 .

The following general conclusions have been drawn from a large ensemble of tested potentials :

- the factor (4) constitutes a good approximation if the number of bound states is large or if the ground and first dipole states are well bound. The accuracy which is reached for $\left\langle r^{2}>\right.$ is of the order of few percent or better.

- the evaluation of $\varphi$ through (4)becomes questionable if the number of bound states is limited or if the ground and first dipole state are weakly bound. This situation occurs for short range potentials. We briefly recall that these potentials decay fast enough to be considered zero beyond a given radius. They are characterized by the existence of critical coupling constants, depending on $\ell$, below which no bound state exists. It is clear, for instance, that the expression (4) loses its meaning if the coupling constant is so low that the lowest dipole level is not bound.

As stated in the introduction, the inequality (1) is saturated for the harmonic oscillator, i.e. it becomes an equality when the first excited state exhausts the sum rule. Consequently it is intuitively legitimate to search for an expression of $\varphi$ based on the fraction of the sum rule carried away by the lowest excited state. It is the purpose of the present work to investigate this question, and to check how far such an expression is dependent on the potential.

Let us denote by

$$
T=2\left[E_{1,1}-E_{1,0}\right]|<1,1| z|1,0>|^{2},
$$

the contribution of the lowest dipole excited state to the sum rule.

A first general property implies $T \leq \varphi$, which is proved in the following way. From Eqs (3) 
and (6), we obtain the following ratio

$$
\frac{T}{\varphi}=4 \pi \frac{|<1,1| r Y_{1,0}|1,0>|^{2}}{<r^{2}>} .
$$

The ground state ms radius can be written

$$
\begin{gathered}
\int_{0}^{\infty} R_{1,0}^{2}(r) r^{4} d r \quad=\int_{o}^{\infty} R_{1,0}^{2}(r) r^{4} d r \int_{\Omega}\left(Y_{1,0}\right)^{2} d \Omega \\
=4 \pi \int R_{1,0}^{2}(r)\left(Y_{1,0}\right)^{2}\left(Y_{0,0}\right)^{2} r^{4} d r d \Omega \\
=4 \pi<1,0\left|r^{2} Y_{1,0}^{2}\right| 1,0>
\end{gathered}
$$

Here, $R_{n, \ell}(r)$ is the radial part of the wave function $\Psi_{n, \ell}(\vec{r})=R_{n, \ell}(r) Y_{\ell, m}(\theta, \phi)$. Then by using the closure, we get

$$
\begin{aligned}
<r^{2}> & =4 \pi \sum_{n}|<n, \ell| r Y_{1,0}|1,0>|^{2} \\
& \geq 4 \pi|<1,1| r Y_{1,0}|1,0>|^{2}
\end{aligned}
$$

It immediately proves that

$$
T \leq \varphi
$$

As before, the equality is reached for the harmonic oscillator. Because of this property, we get a strict lower bound for the ms radius. It reads

$$
<r^{2}>\geq 3|<1,1| z|1,0>|^{2}
$$

As illustrative example, this can be checked with the Coulomb potential for a charge unity. In length units $a_{0}=1$, together with the Bertlmann-Martin bound, it gives

$$
1.665<<r^{2}>=3<4 .
$$

To go beyond this result, it is necessary to calculate explicitly $\varphi$ and $T$ for few potentials. We have started with the power-law potentials (5). Due to scaling properties, the results are independent on the coupling constant $V_{0}$. The calculations have been performed for $-1.5 \leq$ $\alpha \leq 2$. This ensemble has been chosen as a typical set of long range potentials. Note that the case $\alpha=0$ corresponds to the $\ln r$ potential [7]. Apart from the Coulomb potential and the harmonic oscillator, the Schrödinger equation is solved numerically. The results are displayed in fig 1 , where the variations of $T$ and $\varphi$ are shown versus $\alpha$. The two quantities are not proportional, they obey $T \leq \varphi$, the equality being reached asymptotically as $\alpha \rightarrow 2$.

We have completed our study by considering the following typical short range potentials :

- Hulthén : $\quad-V_{0} \frac{e^{-r}}{1-e^{-r}}$

- Yukawa : $\quad-V_{0} \frac{e^{-r}}{r}$

- polynomial : $\quad-V_{0} \frac{1}{(1+r)^{6}}$ 
- Pöschl-Teller : $\quad-V_{0} \frac{1}{\cosh ^{2}(r)}$

- Gaussian : $\quad-V_{0} e^{-r^{2}}$

This sample yields an overview of decays at large $r$ and behaviors near the origin. In the case of the Pöschl-Teller potential, we have added the $\mathrm{D}=1$ case, which is solvable analytically.

In order to exhibit the connection between $T$ and $\varphi$, we found convenient to express the results under the form

$$
\varphi=T^{\nu} .
$$

The exponent $\nu$ is displayed in fig 2 as a function of $T$ for the above sample of potentials. In short range cases, the existence of a critical coupling below which the lowest $\ell=1$ level is not bound makes the calculations near the critical value very delicate. For this reason the curves start at values for which we are confident that the numerical accuracy is of the order of $0.1 \%$.

The spreading of the results is large, and clearly the connection between $\varphi$ and $T^{\nu}$ is depending on the potential. However, this dependence has a well defined structure. The different curves outline a hierarchy among the short range potentials according to the behavior at the origin and the decay at large $r$. A $1 / r$ divergence at $r=0$ yields results close to the ones of the powerlaw potentials, as exemplified by the the Hulthén and the Yukawa potentials. The situation is sensibly different for potential finite at $r=0$. The discrepancy with respect to the power-law case is larger the faster the potential decays.

It may seem paradoxical that the shift of the results goes toward the large $T$ as the decay of the potential becomes faster. Actually, it is due to the fact that for a bound state to exist requires the wave function to be more concentrated inside the potential when the decay is sharper. This produces a larger overlap between the $1 \mathrm{~s}$ and the $1 \mathrm{p}$ wave function for rapidly than slowly decaying potentials.

The curve corresponding to the power-law potentials draws a limit. Denoting by $\nu_{p l}$ the exponent corresponding to the power-law potentials, we obtain the following lower bound

$$
\varphi \geq T^{\nu p l}
$$

which yields

$$
<r^{2}>\geq \frac{3}{2\left(E_{1,1}-E_{1,0}\right)} T^{\nu_{p l}} .
$$

Whereas the actual $\nu$ may differ from $\nu_{p l}$ by a factor 2 or 3 , the impact on $\varphi$ does not exceed $10-15 \%$. Consequently, this lower bound is useful, and it can be combined with the Bertlmann-Martin limit (1). We note also that for power-law potential, $T^{\nu}$ overcomes the difficulty encountered with the correction factor (4) for $-2<\alpha<-1$.

The results for Pöschl-Teller in $D=1$ and $D=3$ are very close. Thus the number of spatial dimensions does not play an important role as long as $D$ remains low. To be more specific, this conclusion is expected to remain valid for the three-body case in the Jacobi coordinates, 
which is equivalent to $D=6$. Because of its analytical solutions, the $D=1$ case offers a possibility to establish the limits $T \rightarrow 0,1$ on firm grounds. To proceed, we replace $r$ by $x$ in the above definition of the Pöschl-Teller potential, and set $V_{0}=s(s+1)$, which is more convenient to express the analytical solutions. The potential strength is thus a function of $s$. The states are labeled by a single quantum number $n$, the ground state wave function is given by $\Psi_{0}(x)=B(1 / 2, s)^{-1 / 2} \cosh ^{-s}(x)$, where $B(1 / 2, s)$ is the Beta function ; the spectrum is given by $E_{n}=-(s-n)^{2}$, which means that $s>1$ in order to bind the lowest dipole state.. We get $[8,9,10]$

$$
\begin{gathered}
<x^{2}>=\frac{1}{2} \zeta(2, s) \leq \frac{1}{2\left(E_{1}-E_{0}\right)}=\frac{1}{2 s-1} \\
\varphi=\frac{2 s-1}{2} \zeta(2, s) \\
T=\frac{s-1}{2}(2 s-1)\left[\frac{\Gamma(s-1 / 2)}{\Gamma(s)}\right]^{4} .
\end{gathered}
$$

Here, $\zeta$ and $\Gamma$ are the Riemann Zeta function and the Gamma function [11], respectively.

From (4), (14) and (15), it can be checked that $\varphi$ and $T \rightarrow 1$ as $s \rightarrow \infty$. In the limit $s \rightarrow 1$, which corresponds to the critical coupling for the $n=1$ state, we found from (14) and (15)

$$
T \rightarrow \frac{s-1}{2} \pi^{2} ; \varphi \rightarrow \frac{\pi^{2}}{12}
$$

To quote numbers, for $s=1.001$ we get $\varphi=0.8225, T=0.0049$ and $\nu=0.0367$. It illustrates the fact that $\nu$ undergoes a vertical tangent as $T \rightarrow 0$, as it can be checked from $\lim _{s \rightarrow 1} d \nu / d T \approx-0.195 /\left[(s-1) \log ^{2}(s-1)\right]$.

\section{$3 \quad$ A bound to the dipole state lifetime.}

The inequality (11) can be inverted, and the measurement of the ground state ms radius provides a upper bound to the dipole transition matrix element. Corollary, it yields a lower bound to the lifetime of the dipole state.

If the correction factor is known, we have

$$
|<1,1| z|1,0>|^{2} \leq \frac{1}{2\left(E_{1,1}-E_{1,0}\right)} \varphi
$$

This is still a strict upper bound. On the other hand, to the extent that $\varphi$ is well approximated by (4), it is tempting to write

$$
|<1,1| z|1,0>|^{2} \leq \frac{1}{2\left(E_{1,1}-E_{1,0}\right)}\left(1-\frac{1}{4}\left[\frac{E_{2,0}+E_{1,0}-2 E_{1,1}}{E_{2,0}-E_{1,0}}\right]^{2}\right) .
$$


To state whether the lower bound character of this relation is ensured requires to know if (4) is actually an upper bound to the true $\varphi$. From systematic studies [3, 4], we found this situation to occur in a large class of potentials, and to be slightly violated in the case of weakly bound systems. This violation is however very small, not exceeding a few percents, and cannot overbalance the inequality (19). Thus we conjecture that (20) is still a upper bound, although it cannot be proved mathematically.

It is interesting to note that (19) yields an upper bound to the transition probability and a lower bound to the life-time of the dipole state in terms of the dipole excitation energy and the energy of the second $\ell=0$ state. If we consider the decay of the dipole state by photon emission, the transition rate is given by [8]

$$
R=\frac{4}{3} \alpha^{3} \frac{\left(E_{1,1}-E_{1,0}\right)^{3}}{\hbar e^{4}}|<1,1| z|1, o>|^{2}
$$

with $\alpha$ the fine structure constant. By using (19) re-establishing the $\hbar$ and $m$ dependence, it leads to

$$
R \leq \frac{2}{3} \alpha^{3} \frac{\left(E_{1,1}-E_{1,0}\right)^{2} \hbar}{m e^{4}} \varphi
$$

and inversely

$$
\tau \geq \frac{3}{2 \alpha^{3}} \frac{m e^{4}}{\left(E_{1,1}-E_{1,0}\right)^{2} \hbar} \frac{1}{\varphi} .
$$

The equality is reached for the harmonic oscillator, as it can be verified easily. The Coulomb case offers an obvious possibility of testing (23). Taking into account $E_{1,1}-E_{1,0}=\frac{3 m e^{4}}{8 \hbar^{2}}$, $\alpha=1 / 137.0037$ and $\frac{\hbar^{3}}{m e^{4}}=2.4210^{-17} \mathrm{sec}$, we get $\tau \geq 0.910^{-9} \mathrm{sec}$, where the actual value is $1.610^{-9} \sec [8]$.

\section{Conclusions.}

We have studied the relationships between the ground state ms radius and the lowest dipole excitation. The problem addresses one particle in a local potential or two particles interacting via a scalar interaction, in $D=3$ space, assuming spherical symmetry. Starting from the Bertlmann-Martin inequality, we have discussed two kinds of correction factors transforming the inequality into approximate or exact expressions. The first one, due to Bertlmann and Martin, requires only the knowledge of the 2 s state energy. It is very efficient for a wide class of potentials.

The second one is based on the sum rule rate exhausted by the lowest dipole excited state $T$. It is expressed as a power-law : $\varphi=T^{\nu}$. This relationship is found dependent on the potential. The results derived for the power-law potentials constitute a limit. In this respect, by measuring $T$ and using this limit, a lower bound to the ms radius is obtained, efficient for the short range potentials. 
The present results show also that the simultaneous measurements of the ground state ms radius, $T$ and the energy of the $1 p$ and 2 s excited states contain a lot of information concerning the potential. They fix characteristics of the potential at least at a qualitative level.

Finally, the inequality (3) expresses a lower bound to the ground state ms radius in terms of the dipole transition matrix element squared. Inverting this relationship produces an upper limit to this square matrix element. Combined with the perturbative treatment of the one photon emission, it produces bounds to the transition probability and the lifetime of the lowest dipole state. To the extend that the Eq. (4) yields a sound approximation to $\varphi$, these bounds require only the knowledge of spectroscopic data.

The present work concerns one- and two-body systems assuming a local potential and spherical symmetry. Its extension to non-local potentials, three-body systems and/or relaxing spherical symmetry deserves attention. However, for this extended cases, the exact solutions are scarce, which makes the investigations much more tedious. Nevertheless they are under consideration for future work.

\section{Acknowledgments}

This work is part of the Franco-Algerian cooperation project CMEP 02 MDU 551.

\section{References}

[1] R.A. Bertlmann and A. Martin, Nucl. Phys. B 168 (1980) 111.

[2] R.A. Bertlmann and A. Martin, Nucl Phys. B 182 (1981) 35.

[3] F.-Z. Ighezou and R.J. Lombard, Ann. of Phys. (NY) 278 (1999) 265.

[4] R. Mezhoud, F.-Z. Ighezou, A. Chouchaoui, A.T. Kerris and R.J. Lombard, Ann. of Phys. (NY) 308 (2003) 143.

[5] S.M. Al-Jaber and R.J. Lombard, J. of Physics A : Math. and Gen. 38 (2005) 1.

[6] A.R. Edmonds, Angular momentum in quantum mechanics, Princeton University Press, Princeton New Jersey, third printing (1974).

[7] R.L. Hall, J. Phys. G : Nucl. Part. Phys. 26 (2000) 981.

[8] see, for instance, S. Flügge, Practical Quantum Mechanics, Springer-Verlag, Berlin Heidelberg, 1971.

[9] A.T. Kerris and R.J. Lombard, Phys. Lett A 319 (2003) 263.

[10] M.M. Nieto, Phys. Rev. A 17 (1978) 1273. 
[11] I.S. Gradshteyn and I.M. Ryzhik, Table of integrals series and products, Academic Press, New York and London, 4th edition (1965).

\section{Captions for figures}

Fig 1 The correction factor $\varphi$ (dashed line) and $T, \mathrm{Eq}(6)$ (solid line) are plotted against $\alpha$ for the power-law potentials $(\mathrm{Eq}(15))$.

Fig 2 The exponent $\nu$ is plotted versus $T$ (see Eq (12)) for the sample of potentials studied in the present work. The solid line is the power-law potentials. Besides potentials quoted in the insert with their symbols, the full triangles are the polynomial, and the full circles the Gaussian potentials, respectively. Note that for $T \geq 0.5$, the Hulthén and Yukawa results coincide with the power-law values. 